\title{
Efficiency and Effectiveness Comparative Analysis of Wide Flange Beams and Cellular Beams in A Case Project United Tractor
}

\author{
Donald Essen and Muhammad Nur Rahman \\ Faculty of Engineering, Mercu Buana University Jakarta, Indonesia \\ donaldessenstmt@gmail.com, mnrahman987@gmail.com
}

\begin{abstract}
United Tractors Company will build a sports center building consist of 3 floors using steel structures. Nowadays, limited land is one of the building development problems. The construction of multi-stored buildings is a solution to the limited land problem. The writer has analyzed beam design with a Wide Flange and Cellular system. Focused on the beam element with construction material in the form of steel with steel quality BJ-37, Fy $240 \mathrm{MPa}$, Fu $370 \mathrm{MPa}$. The strength and efficiency of the use of steel tonnage were also analyzed. E-Tabs software 2016 used to steel beam structure analysis. In this beam design planning refers to the steel structure planning following SNI 1729: 2015, SNI 1727: 2013, AISC 2010, ASCE 7-10, and also AISC Design Guide 31. The results of manual verification show that

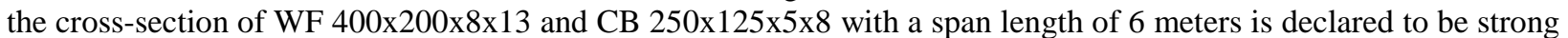
and safe because fill the strong requirements needs to be smaller than the strength of the plan. The efficiency of the use of Cellular Beam was compared to Wide Flange, the longer of Cellular Beam will increase steel tonnage reductions. The percentage reduction in steel weight will continue to increase when the length of steel usage also increases. This concludes that the use of steel length with steel weight reduction will move linearly. Where in this project with a steel beam length of $1439.27 \mathrm{~m}$ reduction of steel tonnage was at $19.5 \%$.
\end{abstract}

Keywords: Wide Flange Beam, Cellular Beam, Comparison

\section{Introduction}

Enthusiasme to exercise the employees of PT. United Tractor encourage management to provide sports facilities and infrastructure. Nowadays, limited land is one of building development problem. The construction of multi-storey buildings is the solution. One of important things in construction of multi-storey building is structural planning. In planning a multi-storey building structure basically must following criteria: Strong in holding planned loads, requirements of serviceability, high durability, accordance with the surrounding environment, economical and easy to maintain [5].

In structural planning, it is necessary to analyze the structure of the reaction caused by axial and lateral forces towards building structure. Structural elements must be designed to able carry an overload of a certain amount, outside of the load expected to occur of normal conditions. Spare capacity is needed to anticipate the possibility of overload factors and undercapacity factors [5]. Based of the problem, this paper analyzes the structures that only review the upper structure, which is the beam element. In this discussion, will analyze the beams with a Wide Flange and Cellular system which will be reviewed regarding the strength and efficiency of the use of steel tonnage. Cellular beam is profile steel I with openings on the body part is which is made by cutting standard profile body parts into 2 parts along the span, then separated, shifted and welded back into new shapes (J.P. Boyer, 1964). The results of this process make the steel profile has higher than the beginning, resulting in the addition of inertia. Increasing inertia will increase the ability of the steel. The economic value of profile I can increase, because with the initial profile I the dimensions are smaller and lighter can be formed into a profile that has a higher height [6]. The steel beam structure analysis is based on SNI 1729: 2015 procedures for planning steel structures for buildings. By using the help of ETABS 2016 computer software for structural analysis.

The rules used are the regulations issued are as follows:

- SNI 1729: 2015 Procedures for planning steel structures for buildings.

- SNI 1727: 2013 Minimum load for building construction planning.

- Steel Design Guide 31 - Castellated and Cellular Beam Design. 


\section{Methodology}

Analysis method used in building structure modeling where the planning used two system, Wide Flange beam system and the Cellular beam system. This research method uses case study analysis method. Case study method is in the form of re-planning with a model that is made in accordance with existing building conditions. The research method used has stages of input, analysis and output. At the input stage will be explained about the structure geometry, dimensions, and specifications of structural elements, the determination of the burden of working with 3-dimensional modeling. The stages of analysis include structural modeling using the 2016 ETABS software. The final stage is the output stage which discusses the strength and efficiency of using steel tonnage. The final result of this plan is to find out what is the tonnage ratio of each design and find out which design is the most efficient if applied to this project.

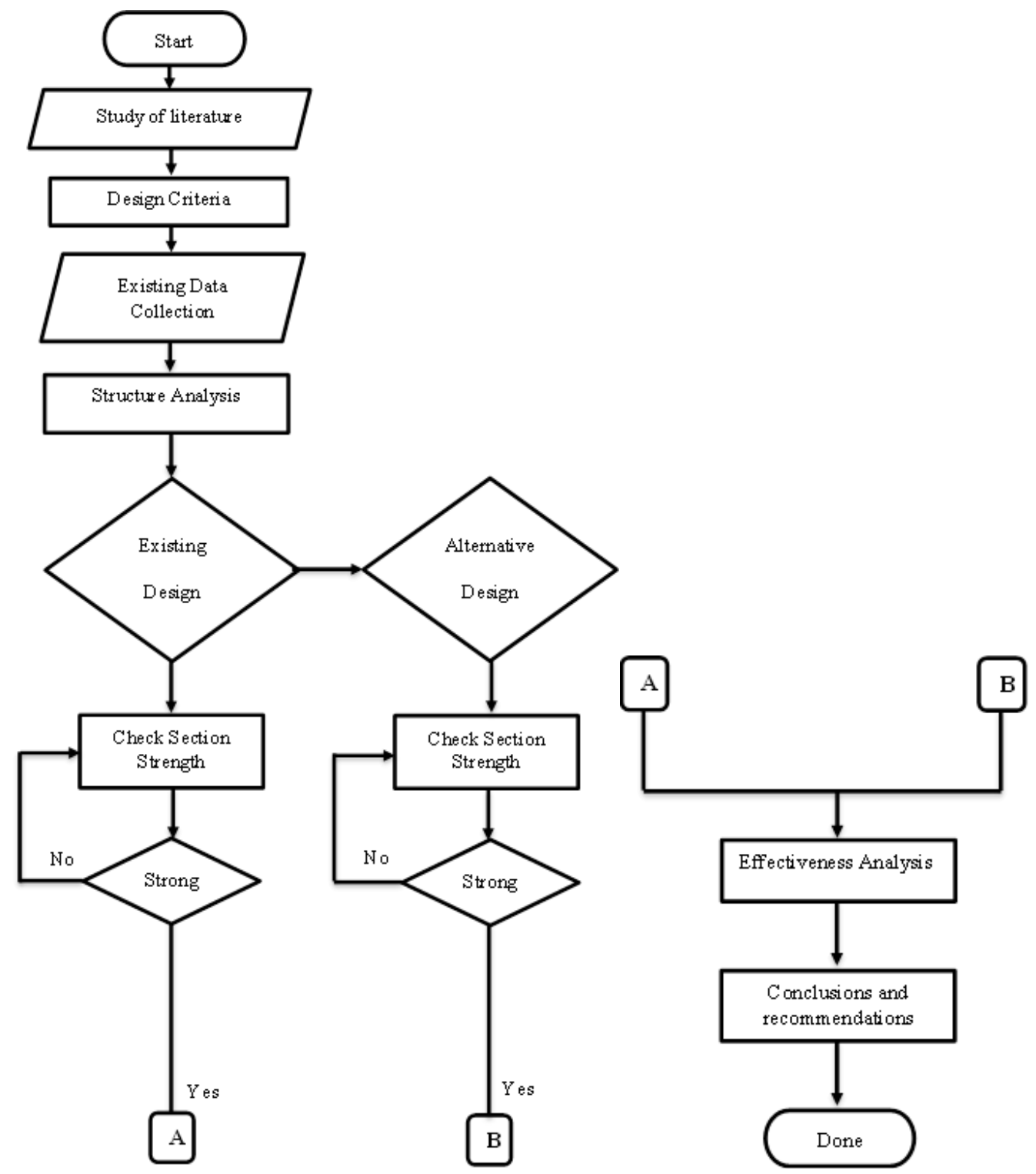

Figure 1. Research Flowchart

Source: Data in research, 2019 


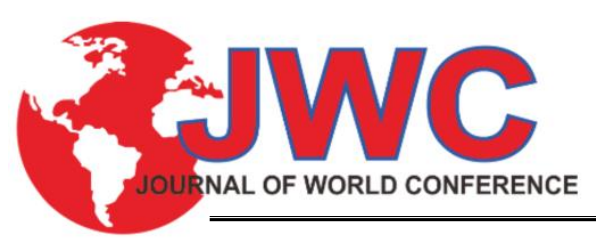

VOLUME 2 | NUMBER 2 | MARCH 2020

Available online at http://proceedings.worldconference.id.

ISSN: 2656-1174 (online)

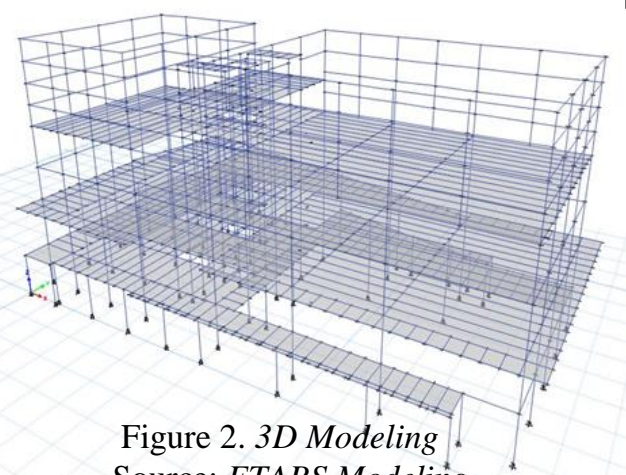

\section{Structural Material}

Concrete Quality

Steel Quality

: $30 \mathrm{Mpa}$

: BJ-37, Fy $240 \mathrm{MPa}$,

Geometric Structure

Number of Floors

Fu $370 \mathrm{MPa}$

Floor Height

: 2 Floors

Building Height

: GF-LT3 (3,5 meter)

Function

: 30 meter

Location

: Sport Center

Type of Soil

: Cakung, North Jakarta

: Medium (SD)

Source: ETABS Modeling

In this study there are 2 types of data, primary data is existing data obtained from the project under study and secondary data from alternative design experiments. The data is then analysed following stages of the research carried out are as follows:

\section{Result and Analysis}

\subsection{Existing Data} follows:

In this case referring to for construction (planning drawings) for the beam size of the Ahemce project is as

Table 1. List of Beam Sizes for Construction

\begin{tabular}{|c|c|c|c|}
\hline Beam Type & Size $(\mathbf{m m})$ & Beam Type & Size $(\mathbf{m m})$ \\
\hline 1 & 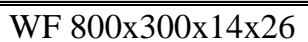 & 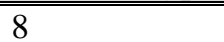 & "WF 400x200x8x13 \\
\hline 2 & WF 700x300x13×24 & 9 & WF $350 \times 175 \times 7 \times 11$ \\
\hline $3 b$ & WF 588x300x12x20 & 10 & WF 300x150x6.5x \\
\hline 4 & WF $600 \times 200 \times 11 \times 17$ & 11 & WF 250x125x6x9 \\
\hline 6 & WF 500x200x10x16 & 12 & WF $200 \times 100 \times 5.5 \times 8$ \\
\hline
\end{tabular}

Source: Ahemce Project Documents 2019

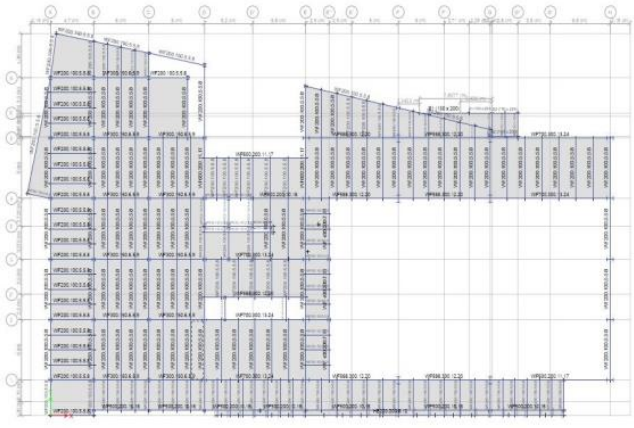

Figure 3. Mezzanine Floor Design

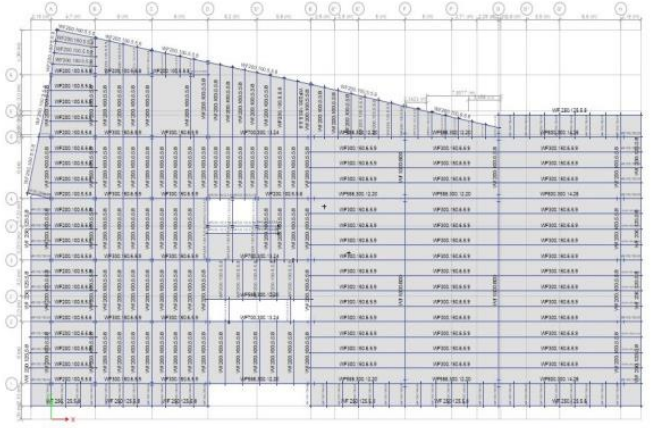

Figure $4.1^{\text {st }}$ Floot Design

Source: Data in research, 2019

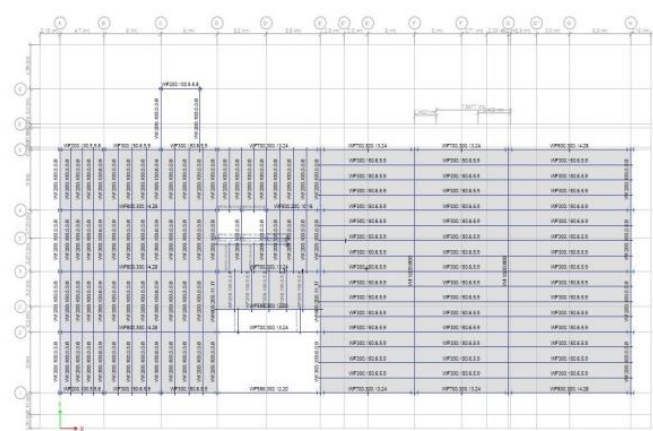

Figure 5. $2^{\text {nd }}$ Floor Design

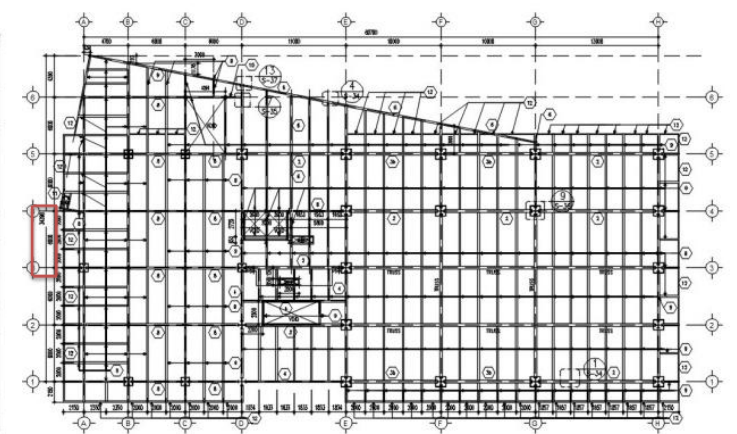

Figure 6 . The position of the beam being observed

Source: Data in research, 2019 


\subsection{Check Section Strength}

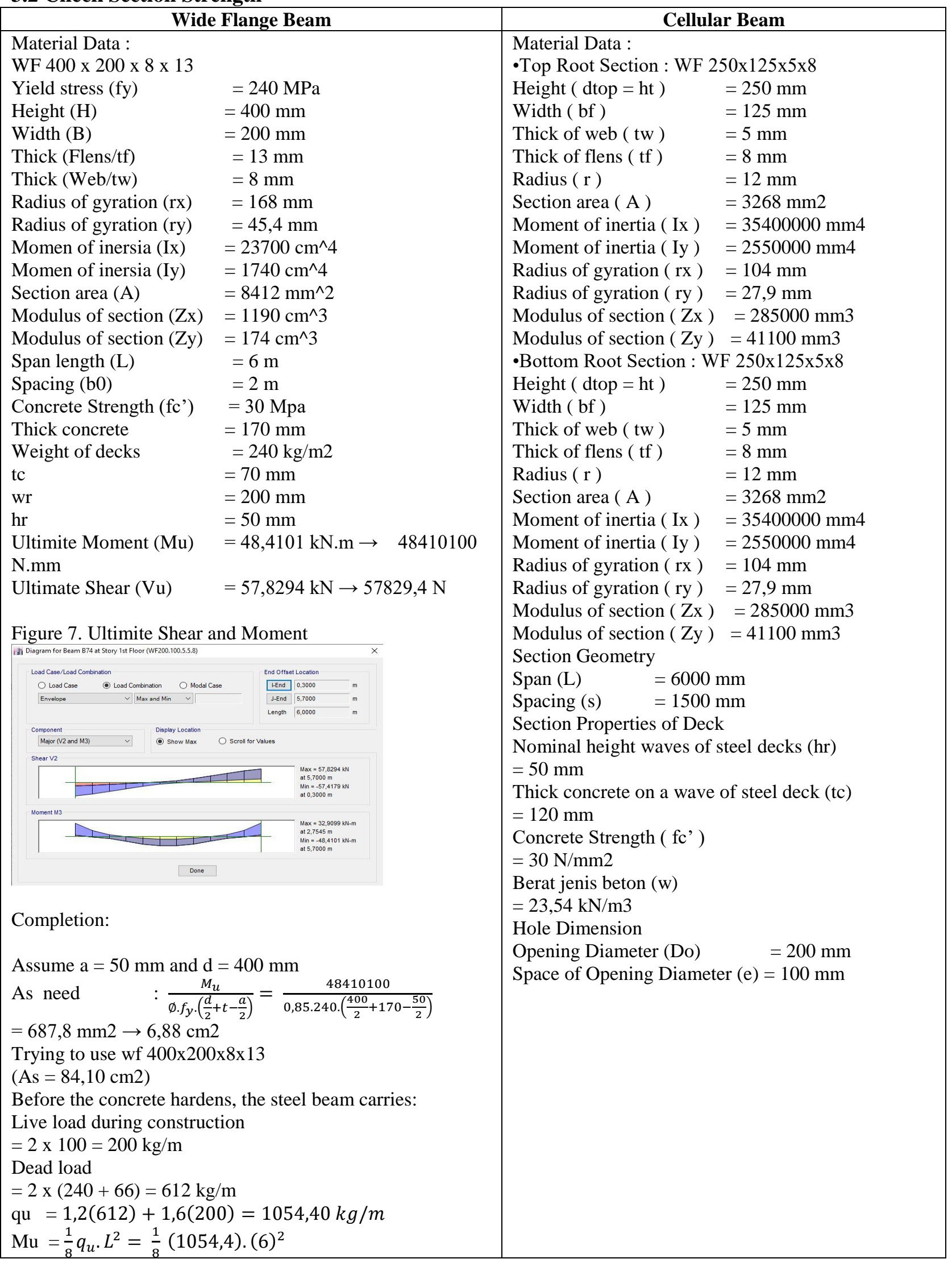




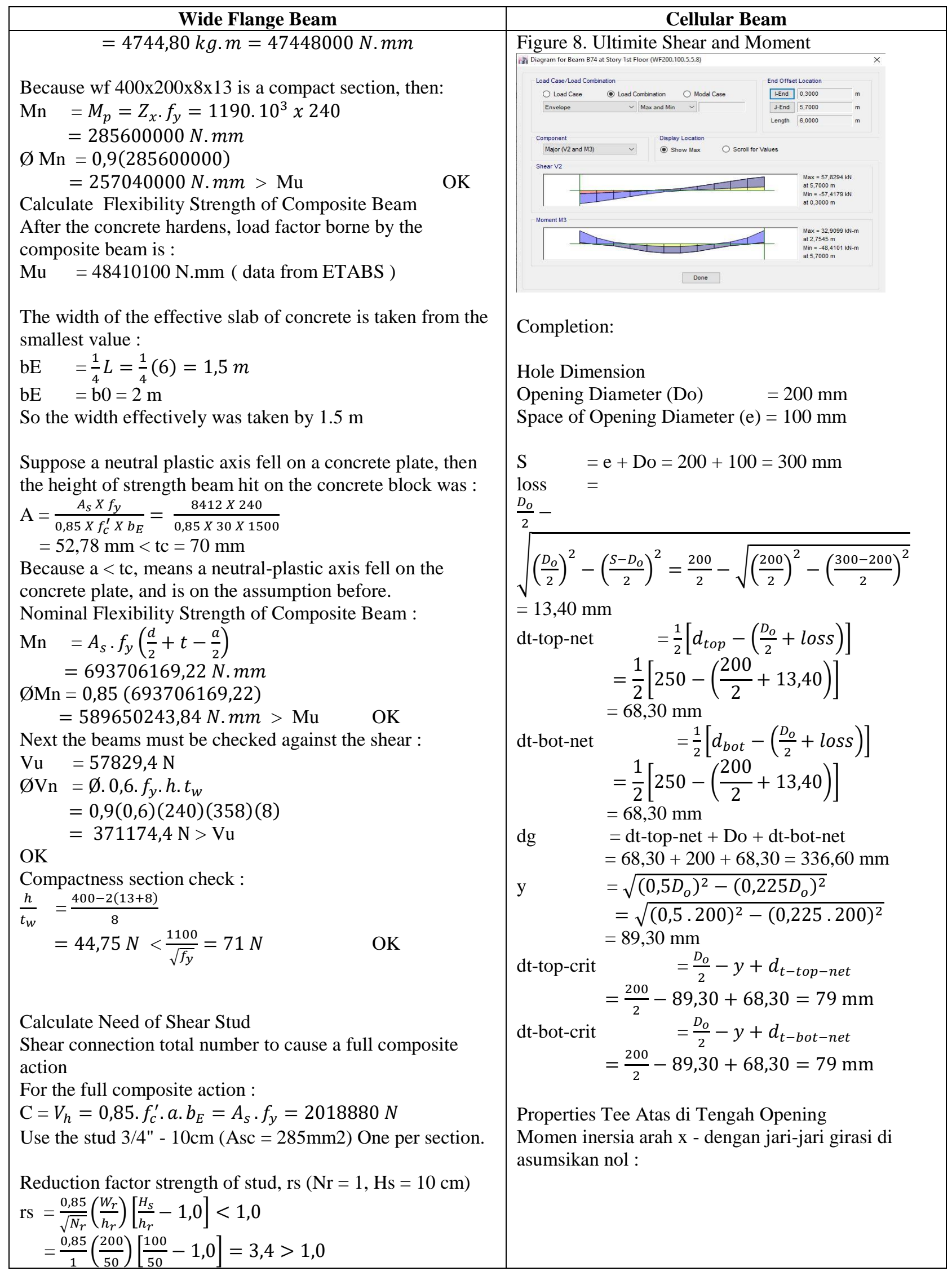


VOLUME 2 | NUMBER 2 | MARCH 2020

Available online at http://proceedings.worldconference.id.

ISSN: 2656-1174 (online)

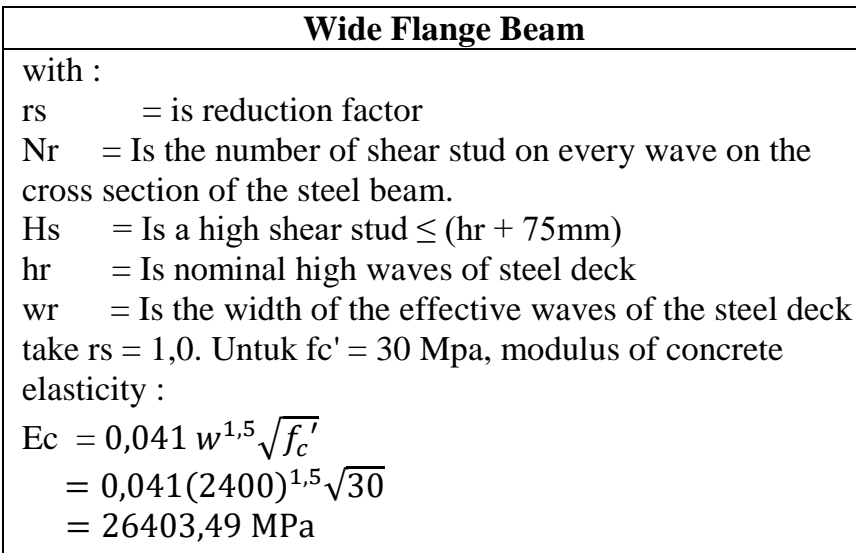

Shear strength a stud:

$$
\begin{aligned}
& \text { Qn }=0,5 \cdot A_{s c} \sqrt{f_{c}^{\prime}} x E_{c} \\
& =0,5(285) \cdot \sqrt{30 \times 26403,49} \\
& =126771,29 \mathrm{~N} \\
& \quad \begin{aligned}
A_{s c} \cdot f_{u} & =285(450) \\
& =128195,31 N>\text { Qn }
\end{aligned}
\end{aligned}
$$

$\mathrm{OK}$

The number of stud required :

$\mathrm{N}=\frac{V_{h}}{Q_{n}}=\frac{2018880}{126771,29}=15,93 \rightarrow 16 \mathrm{stud} \quad($ for $1 / 2$ the span)

For the total lenght it takes 32 stud for the full composite action to occur. If every 2 waves are installed a stud, then the distance between the stud is $2(200)=400 \mathrm{~mm}$, so the number of stud used is as much $(6000 / 400)+1=16$ stud, or 8 stud every $1 / 2$ span.

$\sum \mathrm{Qn} \quad=8(126771,29)=1014170,35 \mathrm{~N}$

Because As. fy $=2018880 \mathrm{~N}>\sum \mathrm{Qn}$, Then there's part of the profile of steel under pressure.

The balance of force that occurred :

$\sum \mathrm{Qn}+\mathrm{Cf} \quad=$ Tmaks $-\mathrm{Cf}$

$1014170,35+\mathrm{Cf}=2018880-\mathrm{Cf}$

$2 \times \mathrm{Cf} \quad=1004709,65$

$\mathrm{Cf} \quad=502354,83 \mathrm{~N}$

The location of the neutral plastic axis calculated from above the flens press is :

$\frac{C_{f}}{b_{f} \times f_{y}}=\frac{502354,83}{200 \times 240}=10,47 \mathrm{~mm}(<\mathrm{tf}=13 \mathrm{~mm})$

Ts location, measured from the underedge of steel flens :

\begin{tabular}{|llll|}
\hline & $\begin{array}{l}\text { Area, A } \\
(\mathrm{cm} 2)\end{array}$ & $\begin{array}{l}\text { Arm, y } \\
(\mathrm{cm})\end{array}$ & $\begin{array}{l}\text { A *y } \\
(\mathrm{cm} 3)\end{array}$ \\
\hline $\begin{array}{l}\text { Concrete } \\
\text { slab }\end{array}$ & 2550 & 8,5 & 21675 \\
WF 400 & 84,12 & 48,5 & 4079,82 \\
\hline & 2634,12 & & 25754,82 \\
\hline
\end{tabular}

$\overline{\mathrm{y}}=\frac{25754,82}{2634}=9,78 \mathrm{~cm}$

Besarnya a dihitung dari persamaan :

\section{Cellular Beam}

Table 2. Properties Tee Atas di Tengah Opening

\begin{tabular}{|c|c|c|c|c|c|}
\hline & Luas (A) & $\begin{array}{c}\text { Timjauan dari } \\
\text { tengah flens y }\end{array}$ & $A y$ & $A y^{2}$ & $I_{0}$ \\
\cline { 2 - 6 } & $\mathbf{m m ^ { 2 }}$ & $(\mathrm{mm})$ & $\mathbf{m m ^ { 3 }}$ & $\mathbf{m m}^{4}$ & $\mathbf{m m}^{4}$ \\
\hline Flens & 1000,00 & 0.0 & 0,0 & 0.0 & 5333,3 \\
\hline Steem & 301,51 & 34,2 & 10296,6 & 106020658,75 & 91362,5 \\
\hline Tee & 1301,51 & & 10296,6 & 106020658,8 & 96695,9 \\
\hline
\end{tabular}

$y_{0-t-t o p-n e t}=\frac{\sum A y}{A_{t-t o p-n e t}}=\frac{10296,6}{1301,51}=7,91 \mathrm{~mm}$

$I_{x-t-t o p-n e t}=$

$\sum I_{0}+\sum A y^{2}-A_{t-t o p-n e t} \cdot y_{0-t-t o p-n e t^{2}}$

$=96695,9+106020658,8-1301,51 \times 7,91^{2}$

$=106035894,7 \mathrm{~mm} 4$

Properties Tee Above on the Critical Section

The moment of inertia in the $\mathrm{x}$ direction - with the radius of gyration assumed to be zero:

Table 3. Properties Tee Above on the Critical Section

\begin{tabular}{|c|c|c|c|c|c|}
\hline & Luas (A) & $\begin{array}{c}\text { Tinjauan dari } \\
\text { tengah flens y }\end{array}$ & $A y$ & $\mathrm{Ay}^{2}$ & $I_{o}$ \\
\cline { 2 - 6 } & $\mathrm{mm}^{2}$ & $(\mathrm{~mm})$ & $\mathrm{mm}^{3}$ & $\mathrm{~mm}^{4}$ & $\mathrm{~mm}^{4}$ \\
\hline Flens & 1000,00 & 0,0 & 0,0 & 0,0 & 5333,3 \\
\hline Steem & 354,99 & 39,5 & 14021,9 & 196613834,29 & 149119,6 \\
\hline Tee & 1354,99 & & 14021,9 & 196613834,3 & 154452,9 \\
\hline
\end{tabular}

$y_{0-t-\text { top-crit }}=\frac{\sum A y}{A_{t-t o p-c r i t}}=\frac{14021,9}{1354,99}=10,35 \mathrm{~mm}$

$I_{x-t-t o p-c r i t}=$

$\sum I_{0}+\sum A y^{2}-A_{t-t o p-c r i t} \cdot y_{0-t-t o p-c r i t}{ }^{2}$

$=154452,9+196613834,3-1354,99 \times 10,35^{2}$

$=196623183,9 \mathrm{~mm} 4$

$$
\begin{aligned}
S_{x-\text { min-t-top-crit }} & =\frac{I_{x-t-t o p-c r i t}}{d_{t-\text { top-crit }}-\left(y_{0-t-t o p-c r i t}+0,5 t_{f-t o p}\right)} \\
& =\frac{196623183,9}{79-(10,35+(0,5 \times 8))} \\
& =3041344,6 \mathrm{~mm} 3
\end{aligned}
$$

\begin{tabular}{|c|c|c|c|c|c|}
\hline & Luas (A) & $\begin{array}{c}\text { Tinjauan dari } \\
\text { tengah flens y }\end{array}$ & Ay & $A y^{2}$ & $I_{o}$ \\
\hline & $\mathrm{mm}^{2}$ & $(\mathrm{~mm})$ & $\mathrm{mm}^{3}$ & $\mathrm{~mm}^{4}$ & $\mathrm{~mm}^{4}$ \\
\hline Flens & 1000,00 & 0,0 & 0,0 & 0,0 & 5333,3 \\
\hline Steem & 301,51 & 34,2 & 10296,6 & 106020658,75 & 91362,5 \\
\hline Tee & 1301,51 & & 10296,6 & 106020658,8 & 96695,9 \\
\hline \multicolumn{2}{|c|}{$y_{0-t-b o t-n e t}$} & \multicolumn{2}{|c|}{$\sum A y$} & \multicolumn{2}{|c|}{$\frac{10296,6}{1301,51}=7,91 \mathrm{~mm}$} \\
\hline \multicolumn{6}{|c|}{$\begin{array}{l}I_{x-t-b o t-n e t}= \\
\sum I_{0}+\sum A y^{2}-A_{t-\text { bot-net }} \cdot y_{0-t-\text { bot-net }}{ }^{2} \\
=96695,9+106020658,8-1301,51 \times 7,91^{2}\end{array}$} \\
\hline
\end{tabular}

Lower Tee Properties in the Middle of Opening The moment of inertia in the $\mathrm{x}$ direction - with the radius of gyration assumed to be zero:

Table 4. Properties Tee Down at the Middle of the Opening 
VOLUME 2 | NUMBER 2 | MARCH 2020

Available online at http://proceedings.worldconference.id.

ISSN: 2656-1174 (online)

$$
\begin{aligned}
& \mathrm{a}=\frac{\sum Q_{n}}{0,85 \times f_{c}^{\prime} \times b_{E}}=\frac{1014170,35}{0,85 \times 30 \times 1500}=26,51 \mathrm{~mm} \\
& \text { Tentukan momen internal terhadap garis kerja Ts : } \\
& \begin{array}{l}
\sum \mathrm{Qn}: \mathrm{Mn} 1=\sum Q_{n}\left(d-\overline{\mathrm{y}}+t_{s}-\frac{a}{2}\right) \\
=1014170,35\left(400-9,78+170-\frac{26,51}{2}\right) \\
=465472715,87 \mathrm{~N} \cdot \mathrm{mm} \\
\mathrm{Cf}: \mathrm{Mn} 2 \quad=C_{f}\left(d-\overline{\mathrm{y}}-\left(\frac{C_{f}}{b_{f} \times f_{y}}\right) / 2\right) \\
=502354,83(400-9,78-5,23) \\
=193401457,14 N \cdot \mathrm{mm}
\end{array} \\
& \begin{array}{r}
\mathrm{Mn}=\mathrm{Mn} 1+\mathrm{Mn} 2=658874173,01 \mathrm{~N} \cdot \mathrm{mm} \\
\varnothing \mathrm{b} . \mathrm{Mn}=0,85(658874173,01) \\
=560043047,06 \mathrm{~N} \cdot \mathrm{mm} \\
=56,00 \text { ton.m }>\mathrm{Mu}(7,44 \text { ton.m) }
\end{array}
\end{aligned}
$$

Jadi, dapat dipasang 16 buah stud 3/4" - $10 \mathrm{~cm}$ dengan jarak $400 \mathrm{~mm}$ ( tiap 2 gelombang dek baja ).

Kontrol Lendutan

Sebelum beton mengeras

$q_{D} \quad=2(240+66)=612 \mathrm{~kg} / \mathrm{m}=6,12 \mathrm{~N} / \mathrm{mm}$

$\begin{aligned} \Delta 1 & =\frac{5 q L^{4}}{384 E I_{S}}=\frac{5 \times 6,12 \times 6000^{4}}{384 \times 200000 \times 23700 \times 10^{4}} \\ & =2,18 \mathrm{~mm}\end{aligned}$

Lendutan akibat beban hidup selama konstruksi

$q_{L} \quad=2(100)=200 \mathrm{~kg} / \mathrm{m}=2 \mathrm{~N} / \mathrm{mm}$

$\Delta 2=\frac{5 q L^{4}}{384 E I_{S}}=\frac{5 \times 2 \times 6000^{4}}{384 \times 200000 \times 23700 \times 10^{4}}$

$$
=0,71 \mathrm{~mm}
$$

Setelah beton mengeras aksi komposit mulai bekerja, momen inersia penampang komposit Itr dihitung sebagai berikut :

$\mathrm{n}=\frac{E_{S}}{E_{c}}=\frac{200000}{26403,49}=7,57 \rightarrow 8$

$\frac{b_{E}}{n}=\frac{1500}{8}=187,50 \mathrm{~mm}$

Luas A ( cm2)

Pelat Beton $=225$

WF $400 \quad=84,12$

$$
\Sigma=309,12
$$

Lengan y $(\mathrm{cm})$

Pelat Beton $=8,5$

$\mathrm{WF} 400 \quad=48,5$

A * y ( cm3)

Pelat Beton $=1912,5$

WF $400 \quad=4079,82$

$\Sigma=5992,32$

Io

Pelat Beton $=2700$

$\mathrm{WF} 400 \quad=23700$

d

Pelat Beton $=10,89$

$\mathrm{WF} 400=-29,11$

Io $+\mathrm{A} * \mathrm{~d}^{\wedge} 2$

\section{Cellular Beam}

$=106035894,7 \mathrm{~mm} 4$

Lower Tee Properties In Critical Sections

The moment of inertia in the $\mathrm{x}$ direction - with the radius of gyration assumed to be zero:

Table 4.5. Lower Tee Properties In Critical Sections

\begin{tabular}{|c|c|c|c|c|c|}
\hline & Luas (A) & $\begin{array}{c}\text { Tinjauan dari } \\
\text { tengah flens y }\end{array}$ & $A y$ & $A y^{2}$ & $I_{o}$ \\
\cline { 2 - 6 } & $\mathrm{mm}^{2}$ & $(\mathrm{~mm})$ & $\mathrm{mm}^{3}$ & $\mathrm{~mm}^{4}$ & $\mathrm{~mm}^{4}$ \\
\hline Flens & 1000,00 & 0,0 & 0,0 & 0,0 & 5333,3 \\
\hline Steem & 354,99 & 39,5 & 14021,9 & 196613834,29 & 149119,6 \\
\hline Tee & 1354,99 & & 14021,9 & 196613834,3 & 154452,9 \\
\hline
\end{tabular}

$y_{0-t-b o t-c r i t}=\frac{\sum A y}{A_{t-b o t-c r i t}}=\frac{14021,9}{1354,99}=10,35 \mathrm{~mm}$

$I_{x-t-b o t-c r i t}=$

$\sum I_{0}+\sum A y^{2}-A_{t-b o t-c r i t} \cdot y_{0-t-b o t-c r i t}{ }^{2}$

$=154452,9+196613834,3-1354,99 \times 10,35^{2}$

$=196623183,9 \mathrm{~mm} 4$

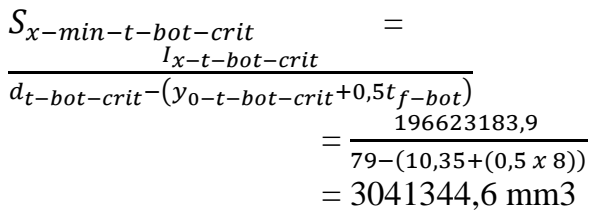

Properties Of Net Section at Middle of Opening The moment of inertia in the $\mathrm{x}$ direction - with the radius of gyration assumed to be zero:

Table 5. Properties Of Net Section at Middle of Opening

\begin{tabular}{|c|c|c|c|c|c|}
\hline & Luas (A) & $\begin{array}{c}\text { Tinjauan dari } \\
\text { bawah Cellular }\end{array}$ & $\mathrm{Ay}$ & $\mathrm{Ay}^{2}$ & $I_{o}$ \\
\cline { 2 - 6 } & $\mathrm{mm}^{2}$ & $(\mathrm{~mm})$ & $\mathrm{mm}^{3}$ & $\mathrm{~mm}^{4}$ & $\mathrm{~mm}^{4}$ \\
\hline Top tee & 1000,00 & 324,7 & 324691,2 & 105424388518 & 106035894,7 \\
\hline Bottom tee & 1000,00 & 11,9 & 11911,3 & 1418795466,81 & 106035894,7 \\
\hline Net Section & 2000,00 & & 336602,5 & 105566268065 & 212071789,3 \\
\hline
\end{tabular}

$y_{\text {steel-net }}=\frac{\sum A y}{A_{\text {net }}}=\frac{336602,5}{2000}=168,30 \mathrm{~mm}$

$I_{\text {steel-net }} \quad=\sum I_{0}+\sum A y^{2}-A_{n e t} \cdot y_{n e t}^{2}$

$=$

$212071789,3+105566268065-$

$2000 \times 168,30^{2}$

$=1,05722 \times 10^{11} \mathrm{~mm}^{4}$

Properties Of Net Section di Bagian Kritikal

Anet-crit $=A_{t-\text { top-crit }}+A_{t-\text { bot-crit }}$

$=1354,99+1354,99$

$=2709,98 \mathrm{~mm}^{2}$

$d_{\text {effec-noncomp }}$

$=d_{g}-\left(0,5 t_{f-t o p}+y_{0-t-t o p-c r i t}\right)-\left(0,5 t_{f-b o t}+\right.$

$\left.y_{0-t-b o t-c r i t}\right)$

$=336,6-((0,5 \times 8)+10,35)-((0,5 \times 8)+$ $10,35)$ 


$$
\begin{aligned}
& \text { Pelat Beton }=546513,23 \\
& \text { WF 400 }=23548336,7 \\
& \\
& \\
& \bar{y} \quad=24094849,93 \\
& =\frac{5992,32}{309}=19,39 \mathrm{~cm}
\end{aligned}
$$

Karena struktur dianggap sebagai balok komposit parsial, maka momen inersia harus direduksi sebagai berikut :

Iefektif $=I_{s}+\left(I_{t s}-I_{s}\right) \sqrt{\frac{\sum \mathrm{Q}_{n}}{C_{f}}}$

$=23700+(24094849,93-23700) \sqrt{\frac{1014170,35}{2018880,0}}$

$=17084407,59 \mathrm{~cm} 4$

Lendutan akibat beban hidup :

$\mathrm{q}=2(488,4)=976,8 \mathrm{~kg} / \mathrm{m}=9,77 \mathrm{~N} / \mathrm{mm}$

$\Delta 3=\frac{5 q L^{4}}{384 E I_{t r}}=\frac{5 \times 9,77 \times 6000^{4}}{384 \times 200000 \times 17084407,59 \times 10^{4}}$

$$
=4,82 \mathrm{~mm}
$$

Lendutan jangka panjang akibat beban mati berupa partisi dihitung sebagai berikut :

$\frac{b}{2 n}=\frac{1500}{2 \times 8}=93,75 \mathrm{~mm}$

Luas A ( $\mathrm{cm} 2)$

Pelat Beton $=112,5$

WF $400=84,12$

$$
\sum=197
$$

Lengan y $(\mathrm{cm})$

Pelat Beton $=8,5$

$\mathrm{WF} 400=48,5$

A * y ( cm3)

Pelat Beton $=956,25$

WF $400=4079,82$

$$
\sum=5036,07
$$

Io

Pelat Beton $=1350$

WF $400=23700$

d

Pelat Beton $=17,11$

$\mathrm{WF} 400=-22,89$

Io $+\mathrm{A} * \mathrm{~d}^{\wedge} 2$

Pelat Beton $=675413,15$

WF $400 \quad=14551208,76$

$\sum=15226621,91$

$\overline{\mathrm{y}} \quad=\frac{5036,07}{197}=25,61 \mathrm{~cm}$

Iefektif ${ }^{\prime}=I_{s}+\left(I_{t s}{ }^{\prime}-I_{s}\right) \sqrt{\frac{\sum \mathrm{Q}_{n}}{C_{f}}}$

$=23700+(15226621,91-23700) \sqrt{\frac{1014170,35}{2018880,0}}$

$=10798947,79 \mathrm{~cm} 4$

\section{$=307,91 \mathrm{~mm}$}

Cellular Beam

Properties Of Net Composite in the Middle of Opening

The moment of inertia in the $\mathrm{x}$ direction - with the radius of gyration assumed to be zero:

Slab transformation:

The effective width of the concrete slab is taken from the smallest value between:

$\mathrm{bE} \quad=\frac{1}{4} \cdot L=\frac{1}{4} \cdot 6000=1500 \mathrm{~mm}$

$\mathrm{bE} \quad=\mathrm{bo}=1500 \mathrm{~mm}$

$\mathrm{bE}$ dipakai $\quad=1500 \mathrm{~mm}$

Calculating the value " $n$ "

$\mathrm{n}=\frac{E_{S}}{E_{c}}=\frac{200000}{4700 \sqrt{f_{c^{\prime}}}}=\frac{200000}{4700 \sqrt{30}}=7,77$

Plate equivalent effective width

$\frac{b_{E}}{n}=\frac{1500}{7,77}=193,07 \mathrm{~mm}$

Table 6. Properties Of Net Composite in Middle of Opening

\begin{tabular}{|c|c|c|c|c|c|}
\hline & Luas (A) & $\begin{array}{c}\text { Tinjauan dari } \\
\text { bawah Cellular }\end{array}$ & $A y$ & $\mathrm{Ay}^{2}$ & $I_{o}$ \\
\cline { 2 - 6 } & $\mathrm{mm}^{2}$ & $(\mathrm{~mm})$ & $\mathrm{mm}^{3}$ & $\mathrm{~mm}^{4}$ & $\mathrm{~mm}^{4}$ \\
\hline $\begin{array}{c}\text { Transformed } \\
\text { Slab }\end{array}$ & 32822,27 & 421,6 & 13837954,2 & 1914889766732322 & 464982,2 \\
\hline Net Section & 2000,00 & 168,3 & 336602,5 & 113301270189,22 & 105721689219,3 \\
\hline $\begin{array}{c}\text { Composite } \\
\text { Section }\end{array}$ & 34822,27 & & 14174556,7 & 191602277943421 & 105722154201,5 \\
\hline
\end{tabular}

$y_{t r-n e t}=\frac{\sum A y}{A_{t r}-n e t}=\frac{14174556,7}{34822,27}=407,1 \mathrm{~mm}$

$I_{\text {comp-net }}=$

$\sum I_{0}+\sum A y^{2}-A_{\text {tr-net }} \cdot y_{\text {tr-net }}{ }^{2}$

$=105722154201,5+191602277943421-$

$34822,27 \times 407,1^{2}$

$=191702230283235 \mathrm{~mm} 4$

Global and Local Style in the First Opening Top Tee First opening location:

$\mathrm{x}=\mathrm{e}+0.5 \mathrm{Do}=100+0.5 \times 200=200 \mathrm{~mm}$

Global shear force:

The shear force that exists at the distance $\mathrm{x}$

$\mathrm{Vu}$ at a distance

$\mathrm{X}=57418,17 \mathrm{~N}$

Gaya geser nominal pada beton :

$$
\begin{aligned}
& \mathrm{Vnc}=3(h r+t c) \cdot(t c) \cdot\left(4 \sqrt{f_{c}^{\prime}}\right) \\
& =3(50+120) \cdot(120) \cdot(4 \sqrt{30}) \\
& =1340824,82 \mathrm{~N} \\
& \mathrm{Vc}=\emptyset_{c v} \cdot V_{n c}=0,75 \times 1340824,82 \\
& =1005618,62 \mathrm{~N} \\
& \text { Vnet }=V_{u}-V_{c}=57418,17-1005618,62 \\
& =-948200,45 \mathrm{~N}
\end{aligned}
$$




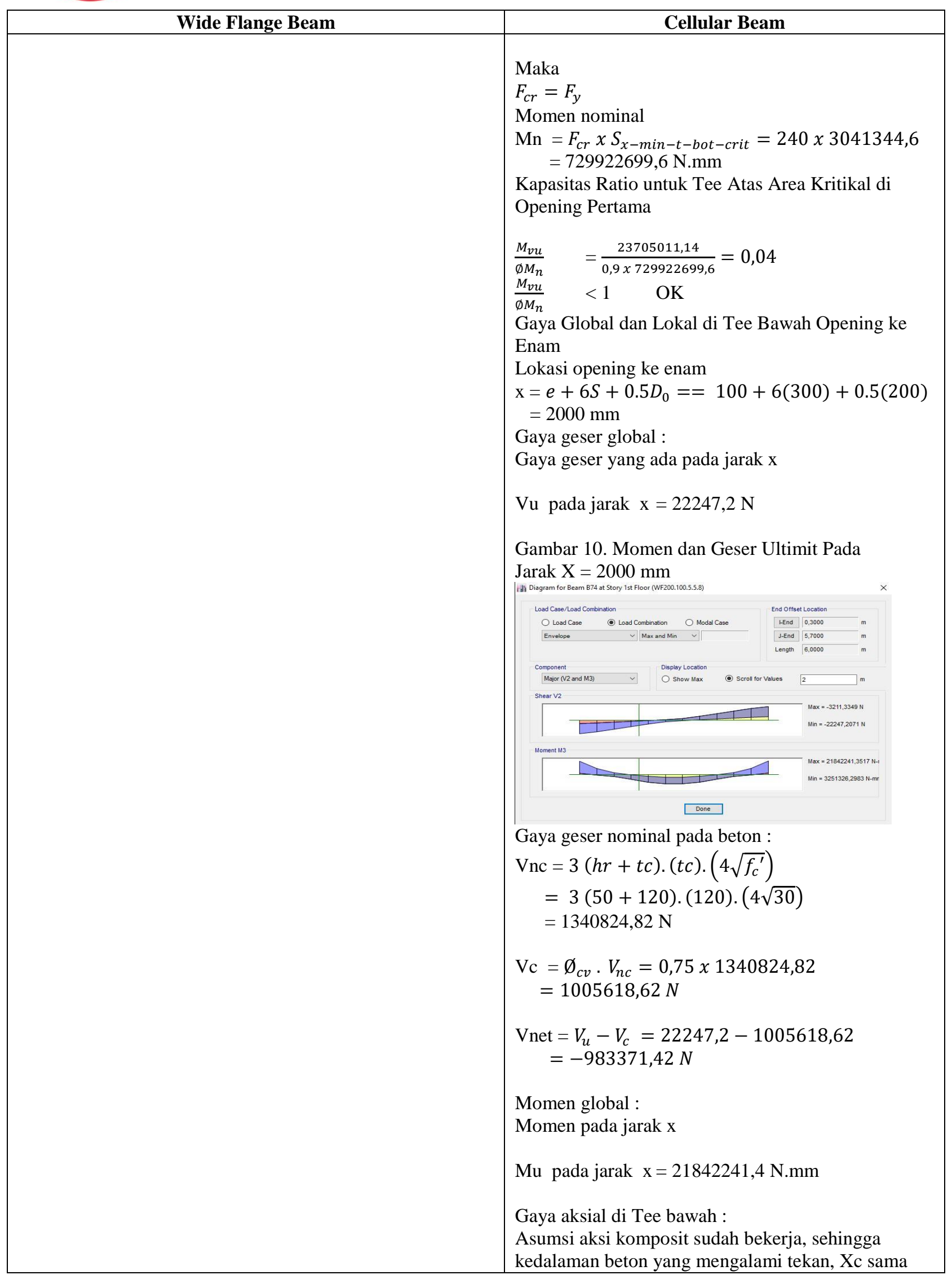




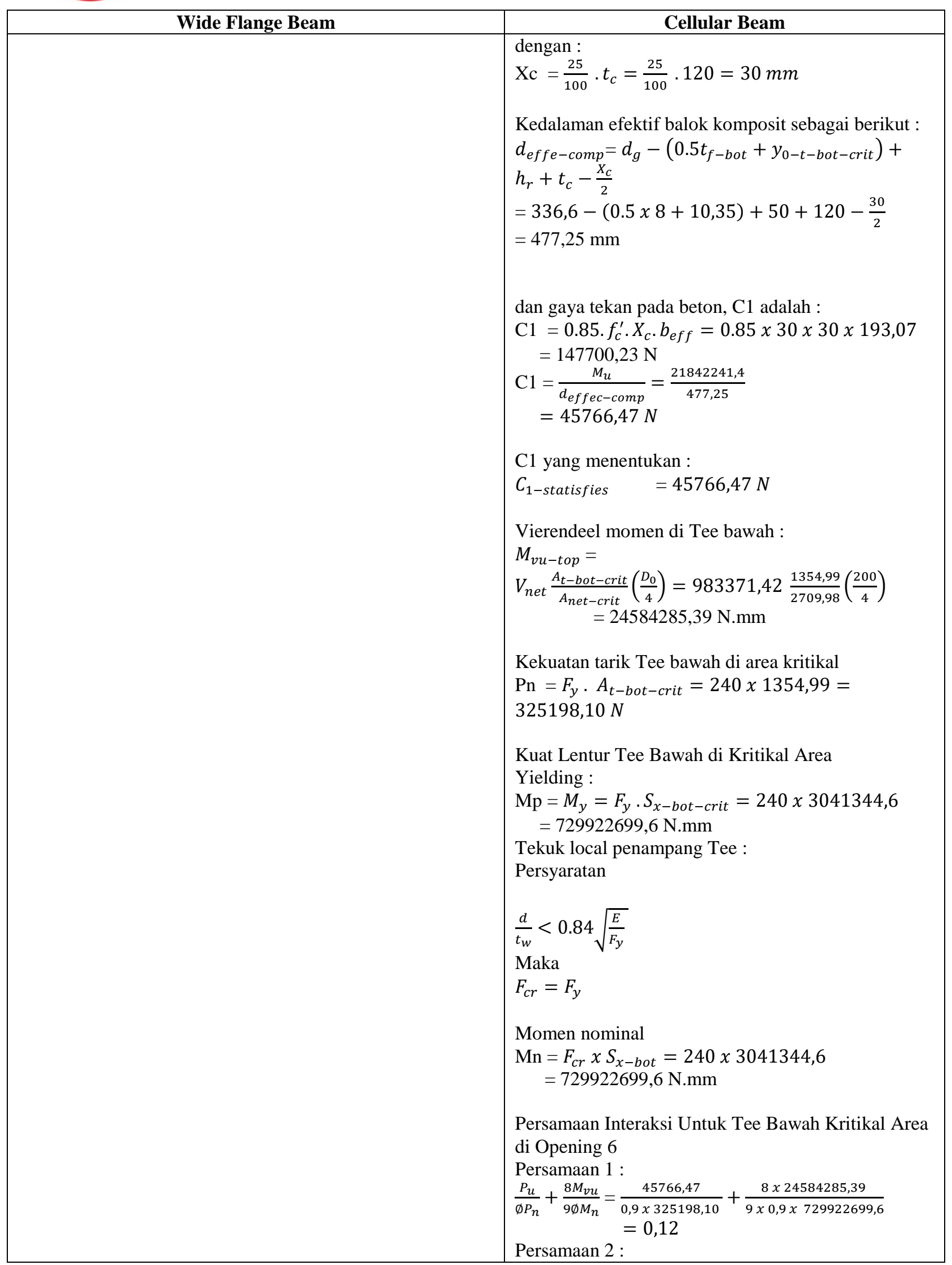




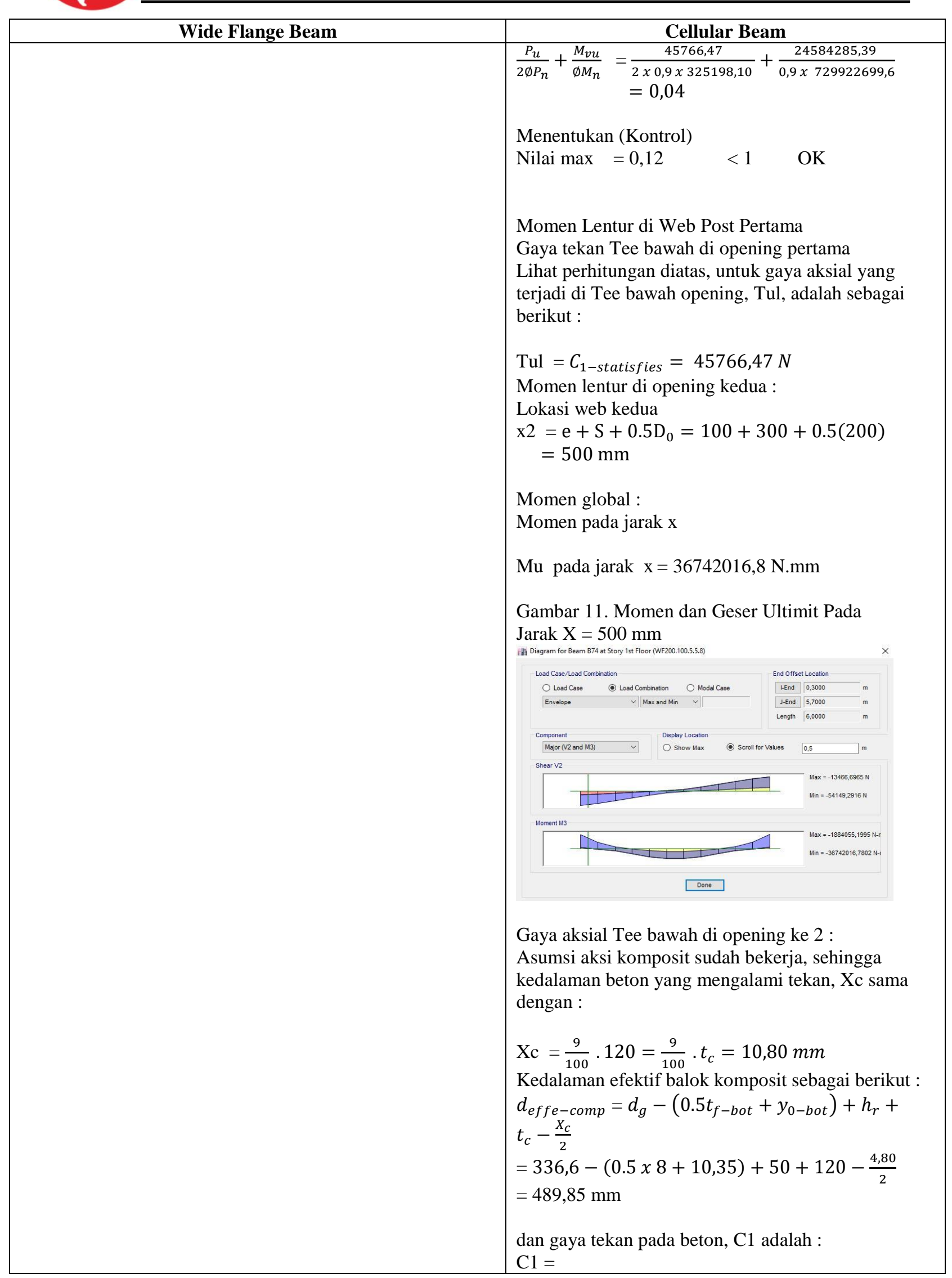




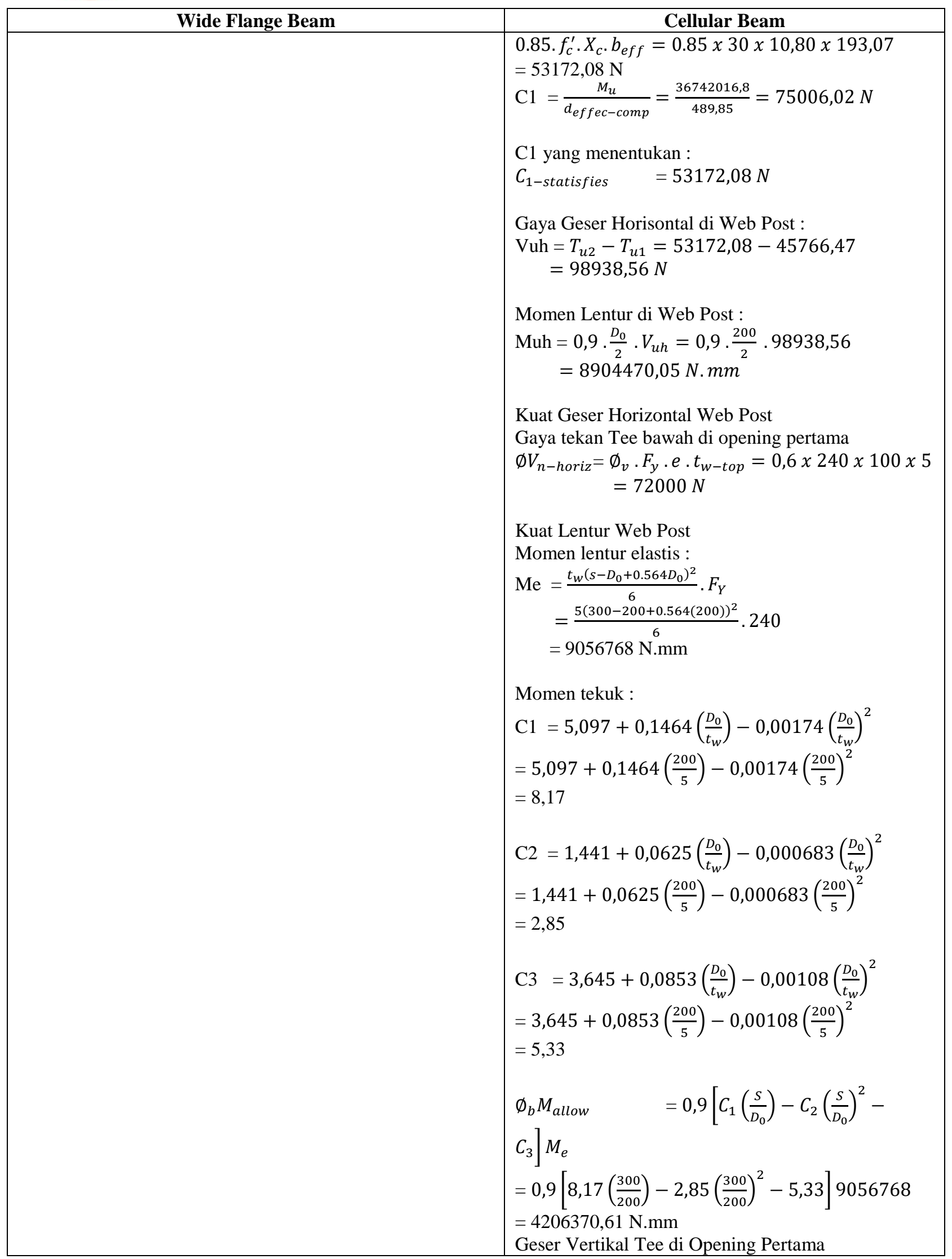




\section{Cellular Beam}

Lokasi opening pertama

$\mathrm{x}=e+0.5 D_{0}=100+0.5(200)=200 \mathrm{~mm}$

Geser :

Geser Ultimit

$\mathrm{Vu}=57418,17 \mathrm{~N}$

$V_{u-\text { top-tee }}=\frac{A_{t-\text { top }- \text { net }}}{A_{\text {net }}} V_{u}=\frac{1000}{2000} \cdot 57418,17$ $=28709,09 \mathrm{~N}$

$V_{u-\text { bottom-tee }}=\frac{A_{t-\text { bottom-net }}}{A_{\text {net }}} V_{u}=\frac{1000}{2000} \cdot 57418,17$ $=28709,09 \mathrm{~N}$

Kuat Geser Nominal :

Persyaratan

$\frac{h}{t_{w}}=\frac{d_{t-t o p-n e t}}{t_{w-t o p}} \leq 1,1 \sqrt{k_{v} \frac{E}{F_{y}}} \quad$ maka $\quad C_{v 2}=$ 1.0

$\frac{h}{t_{w}}=\frac{d_{t-t o p-n e t}}{t_{w-t o p}}=\frac{68,3}{5}=13,66$

$1,1 \sqrt{k_{v} \frac{E}{F_{y}}}=1,1 \sqrt{1,2 \times \frac{200000}{240}}=34,8$

$\emptyset_{v} V_{n-t e e-t o p}=\emptyset_{v} \cdot 0,6 \cdot F_{y} \cdot\left(d_{t-t o p-n e t} \cdot t_{w-t o p}\right) \cdot C_{v 2}$ $=1 \times 0,6 \times 240 \times(68,3 \times 5) \times 1$

$=49176,9 \mathrm{~N}$

Persyaratan

$\frac{h}{t_{w}}=\frac{d_{t-b o t-n e t}}{t_{w-b o t}} \leq 1,1 \sqrt{k_{v} \frac{E}{F_{y}}} \quad$ maka $\quad C_{v 2}=$ 1.0

$\frac{h}{t_{w}}=\frac{d_{t-\text { bot-net }}}{t_{w-b o t}}=\frac{68,3}{5}=13,66$

$1,1 \sqrt{k_{v} \frac{E}{F_{y}}}=1,1 \sqrt{1,2 \times \frac{200000}{240}}=34,8$

$\emptyset_{v} V_{n-t e e-b o t}=\emptyset_{v \cdot 0,6 . F_{y}}\left(d_{t-b o t-n e t} \cdot t_{w-b o t}\right) \cdot C_{v 2}$ $=1 \times 0,6 \times 240 \times(68,3 \times 5) \times 1$

$=49176,9 \mathrm{~N}$

Geser Vertikal Pada Bagian Kotor Geser

$\mathrm{Vu}=57829,14 \mathrm{~N}$

Gambar 12. Momen dan Geser Ultimit 

ISSN: 2656-1174 (online)

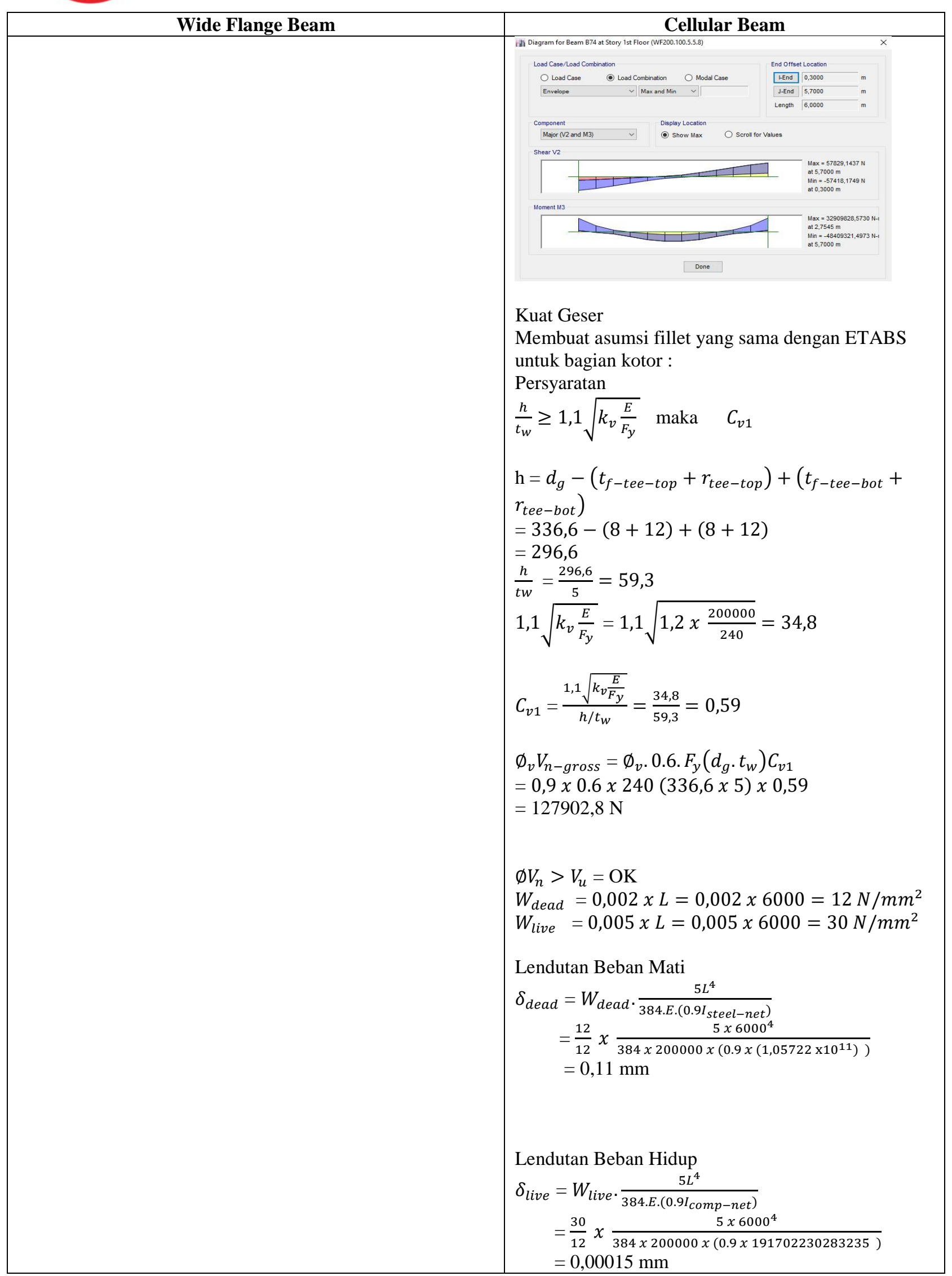




\begin{tabular}{|l|l|}
\hline Wide Flange Beam & Cellular Beam \\
\hline & $\begin{array}{l}\text { Lendutan total yang terjadi : } \\
\delta_{\text {dead }}+\delta_{\text {dead }}=0,11+0,00015 \\
=0,11015 \mathrm{~mm} \leq \frac{L}{280}=21,42 \mathrm{~mm} \\
\end{array}$ \\
\hline
\end{tabular}

Further calculations on each cellular beam type can be seen in the appendix, while the size (type) of the beam used is as follows:

Table 7. List of Cellular Beam Size

\begin{tabular}{|l|l|l|l|l|l|}
\hline $\begin{array}{c}\text { Nama } \\
\text { Balok }\end{array}$ & \multicolumn{1}{|c|}{$\begin{array}{c}\text { Ukuran } \\
(\mathrm{mm})\end{array}$} & $\begin{array}{c}\text { Tinggi } \\
(\mathrm{mm})\end{array}$ & \multicolumn{1}{|c|}{$\begin{array}{c}\text { Lebar } \\
(\mathrm{mm})\end{array}$} & \multicolumn{1}{|c|}{$\begin{array}{c}\text { Lubang Cellular } \\
\varnothing\end{array}$} & $\begin{array}{l}\text { Spasing } \\
\varnothing\end{array}$ \\
\hline $\mathrm{A}$ & $250 \times 125 \times 5 \times 8$ & 334.6 & 124 & 200 & 300 \\
\hline $\mathrm{B}$ & $300 \times 150 \times 5.5 \times 8$ & 421.2 & 150 & 240 & 360 \\
\hline $\mathrm{C}$ & $350 \times 175 \times 6 \times 9$ & 471.2 & 175 & 280 & 420 \\
\hline $\mathrm{D}$ & $350 \times 175 \times 7 \times 11$ & 471.2 & 175 & 280 & 420 \\
\hline $\mathrm{E}$ & $346 \times 174 \times 6 \times 9$ & 467.2 & 174 & 280 & 420 \\
\hline F & $400 \times 200 \times 7 \times 11$ & 538 & 200 & 320 & 480 \\
\hline G & $400 \times 200 \times 8 \times 13$ & 538 & 200 & 320 & 480 \\
\hline H & $450 \times 200 \times 9 \times 14$ & 605.9 & 200 & 360 & 540 \\
\hline I & $500 \times 200 \times 10 \times 16$ & 673.2 & 200 & 400 & 600 \\
\hline J & $600 \times 200 \times 11 \times 17$ & 816.5 & 200 & 500 & 750 \\
\hline K & $600 \times 300 \times 12 \times 20$ & 807 & 300 & 480 & 720 \\
\hline
\end{tabular}

(Source : Author)

\section{Conclusions \& Suggestions}

\subsection{Conclusions}

Based on the results of the research it can be concluded that:

Table 8. Beams Checked Against The Shear

\begin{tabular}{ccccc}
\hline & & \multicolumn{3}{c}{ Chek } \\
No & Steel Type & \multicolumn{2}{c}{ Shear $(\varnothing V n>V u)$} \\
& & $V u$ & $\varnothing V n$ & Status \\
\hline 1 & WF $400 \times 200 \times 8 \times 13$ & 57829,4 & 371174,4 & OK \\
2 & CB 250x125x5x8 & 57829,14 & 127902,85 & OK \\
\hline
\end{tabular}

Table 9. Beams Checked Against The Nominal Flexibility Strength

\begin{tabular}{|c|c|c|c|c|}
\hline \multirow{3}{*}{ No } & \multirow{3}{*}{ Steel Type } & \multicolumn{3}{|c|}{ Chek } \\
\hline & & \multicolumn{3}{|c|}{ Moment $(\varnothing M n>M u)$} \\
\hline & & $M u$ & $\varnothing M n$ & Status \\
\hline 1 & WF 400x200x8x13 & 48410100 & 589650243,8 & $\mathrm{OK}$ \\
\hline 2 & CB $250 \times 125 \times 5 \times 8$ & 48410100 & 656930429,7 & $\mathrm{OK}$ \\
\hline
\end{tabular}

From the results of manual calculation verification, it shows that the cross section of WF 400x200x8x13 and CB $250 \times 125 \times 5 \times 8$ with a span length of 6 meters is declared strong and safe because it fill the necessary strong requirements, smaller than the strength of the plan. 
Figure 13. Efficiency Graph of Wide Flange And Cellular Beams

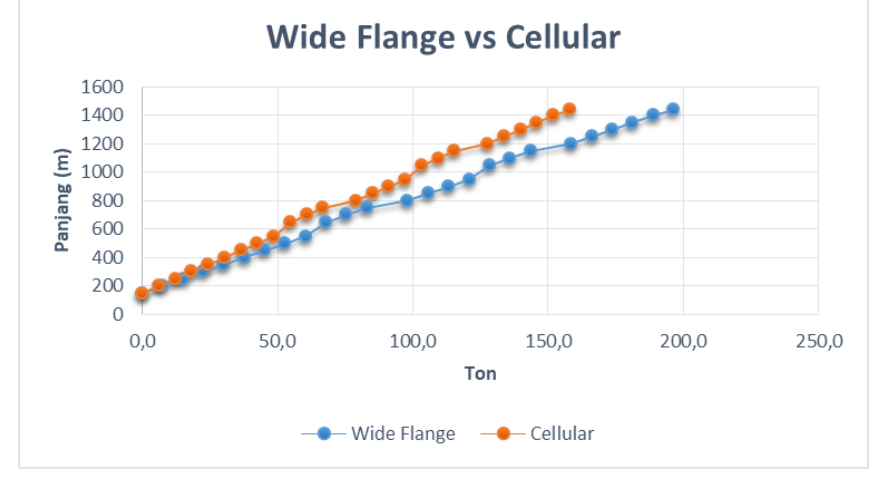

From the graph above it can be seen the efficiency of using Cellular Beam compared to Wide Flange. Where the longer $(\mathrm{m})$ use of Cellular Beam, will increases steel tonnage reduction.

Figure 14. Graph Percentage Of Steel Weight Reduction

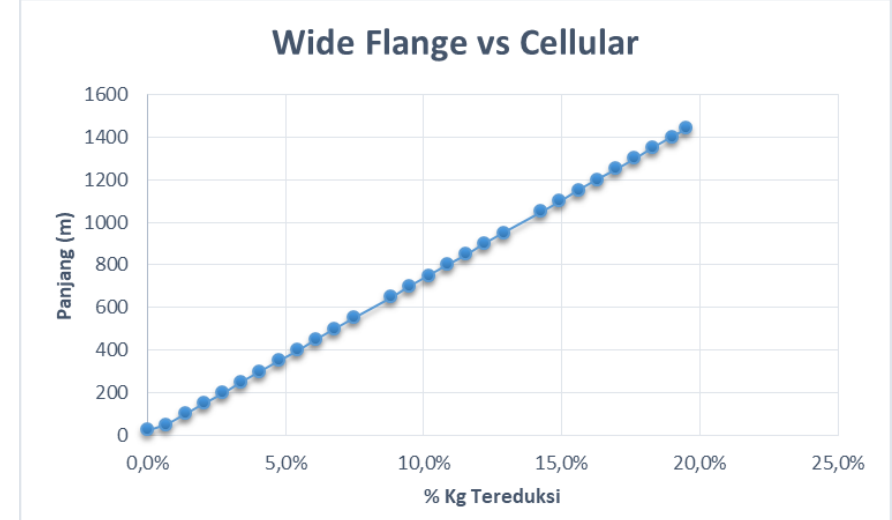

From the graph above explains the percentage reduction of steel weight will continue to increase when the length of steel usage also increases. This concludes that the use of steel length with steel weight reduction will move linearly. Where in this project with a steel beam length of $1439.27 \mathrm{~m}$ reduction of steel tonnage was at $19.5 \%$.

\subsection{Suggestions}

Based on the results that have been researched can be given suggestions, including:

1. In calculating the analysis in this study using the standard burdens of existing regulations. To get a more accurate structural analysis, it is recommended to use the burdens that have been imposed on the structure design by the planner (consultant).

2. For further research, analysis of beams can use other types of blocks such as asymmetrical cellular or castellated. So that we will get a broader conclusion than other alternatives.

\section{References}

[1] Fares, S., Coulson, J., \& Dinehart, D. (2016). AISC design guide 31 castellated and cellular beam design. American Institute of Steel Construction, USA.

[2] Nasional, B. S. (2013). Beban Minimum Untuk Perancangan Bangunan Gedung dan Struktur Lain SNI 1727: 2013. Jakarta: BSN.

[3] Nasional, B. S. (1989). Pedoman Perencanaan Pembebanan Untuk Rumah dan Gedung: SNI 1727: 1989. Jakarta, Indonesia.

[4] Nasional, B. S. (2015). SNI 1729-2015: Spesifikasi untuk Bangunan Gedung Baja Struktural. Jakarta: Badan Standardisasi Nasional, 289.

[5] Setiawan, Agus. (2013) : Perencanaan Struktur Baja dengan Metode LRFD, Edisi ke-2, Erlangga, Jakarta

[6] Situmorang, A. H. M. (2017). Analisa Perbandingan Cellular Beam dan Honeycomb Beam dengan Program 
Ansys. Jurnal Teknik Sipil USU, 7(1).

\section{Biographies}

Donald Essen was a professor who, today, actively teaches at the Mercubuana University Jakarta with a concentrate on structure. He got a bachelor's degree in civil engineering from the bandung institute of technology in 2002, an Master' degree in engineering specialist from the bandung institute of technology in 2007. He is a member of the Indonesian society of civil and engineers, registered as haki professional engineer, also Class A professional for construction engineering from Dinas Pengawasan dan Penertiban Bangunan DKI Jakarta (DPPB). Currently the director of ESK Enjiniring Company.

Muhammad Nur Rahman was born in Yogyakarta, a special region of yogyakarta on August 9th, 1997. After graduation a vocational high school education in building engineering department continued his undergraduate civil engineering education at mercubuana university jakarta in 2016 to 2020 by compiling the final project is "Efficiency and effectiveness comparative analysis of wide flange beams and cellular beams in a case project united tractor", also active as a staff in building constuction project. 